necrosis factor (TNF) therapy in patients with refractory $C D$, who have lost response to standard dose treatment.

Methods A retrospective interrogation of our local inflammatory bowel disease database at Queen Elizabeth Hospital, Woolwich was undertaken to identify all patients who had had either dose-doubling or decreased dosing intervals of their anti-TNF therapies. Clinic letters, hospital notes, biochemical, endoscopic and radiological data were recorded and disease severity scores calculated using the Harvey-Bradshaw Index. We present our data describing efficacy and safety of these biologic agents at higher dosage.

Results A total of sixteen patients were in our study, 9(56\%) female and $7(44 \%)$ male and the mean age was 38 years (range $21-$ 68 years). Median disease duration was 6 years (range $2-13$ years). There were 12 patients who were initially started on Infliximab while 4 had Adalimumab as their initial biologic therapy. 11(69\%) patients had dose-doubling and $5(31 \%)$ patients had decreased dosage intervals due to secondary loss of response to initial anti-TNF at standard dosage regimen. Early response to dose-escalation was experienced by $9 / 16(56 \%)$ patients while $7 / 16(44 \%)$ patients failed to respond to alteration in anti-TNF therapy regimen. Of the nine patients who initially showed response to intensified regimens, sustained clinical remission was maintained in $5(31 \%)$ patients at 12 months and this cohort was successfully weaned of biologic therapy. There was secondary loss of response in $4(25 \%)$ patients after median of 7.5 months (range 6-10) at this intensified regimen. No adverse effects were noted in our cohort of patients at this intensified regimen.

Conclusion Our experience of managing CD patients who have failed on their initial standard dose biological therapies has showed that there is certainly value in trialling either increased dosage or decreased dosing intervals of anti-TNF agent. Five patients achieved sustained clinical response and 4 patients had a median further 7 months of disease control prior to relapse. Higher anti-TNF dosage appears to be well tolerated and safe in CD. Of the patients who did fall in the latter two groups, there was a tendency towards reducing the dosing intervals as the more successful strategy above dose-doubling; however this was not statistically significant.

Disclosure of Interest None Declared.

\section{PTH-091 TO EVALUATE THE EFFICACY AND SAFETY OF 6-THIOGUANINE THERAPY IN PATIENTS WITH INFLAMMATORY BOWEL DISEASE - A DGH EXPERIENCE}

doi:10.1136/gutjnl-2013-304907.578

1."R Saleem, 'A Gera, 'A Paolino, 'A Loganayagam. ' Gastroenterology, Queen Elizabeth Hospital, Woolwich., London, UK

Introduction Conventional thiopurines (Azathioprine/6-Mercaptopurine) are considered to be safe and effective in the treatment of inflammatory bowel disease (IBD). Unfortunately more than 50\% of patients discontinue thiopurine therapy, mainly due to the development of adverse events or therapy resistance ${ }^{1}$. In recent years, 6-thioguanine (TG) has been used as an alternative thiopurine in IBD patients failing to tolerate or to respond to conventional thiopurine therapy. The aim of this study was to evaluate the tolerability, safety and efficacy of 6-thioguanine in the treatment of IBD patients in a District General Hospital (DGH).

Methods A retrospective database analysis was performed on all IBD patients who had previously failed to respond or to tolerate conventional thiopurine therapy and were subsequently treated with TG at $20 \mathrm{mg}$ once daily. Rates and reasons for treatment failure were assessed. Clinical features, laboratory values, abdominal imaging and endoscopic remission rates were evaluated.

Results Total of twelve patients received TG and median treatment duration was 8 months (range 1-12). There were 7(58\%) female and $5(42 \%)$ male. Mean age was 37 years (range 19-62).
$6 / 12(50 \%)$ patients had Ulcerative Colitis (UC) and 6/12(50\%) had Crohn's Disease (CD). Indications for initiation of 6-thioguanine therapy were conventional thiopurine intolerance 10(83\%) and non-response to treatment $2(17 \%)$.

Two patients stopped taking treatment within first month due to fear of side effects; one of them wanted to become pregnant. Of 10 patients two failed TG therapy: one due to adverse event (hair loss) and other due to therapy failure. 4/10(40\%) patients had partial response, having occasional mild flare ups while further $6 / 10(60 \%)$ patients remained in sustained clinical remission at 6 months on treatment, although one of the patient did not turn up to follow up appointment following 6 months of therapy. Tolerability and efficacy rates were similar in both UC and CD. All patients were closely monitored and no abnormality in liver function tests detected.

Conclusion Our study showed that TG was well tolerated in this selected group of difficult to treat patients. In addition, the use of small dose $20 \mathrm{mg}$ daily of TG appears to be relatively safe in IBD patients who failed conventional thiopurine therapy. Well designed prospective trials are required to further evaluate the safety and efficacy of 6-thioguanine.

Disclosure of Interest None Declared.

\section{REFERENCE}

1. Jharap B, Seinen ML, De Boer NK, et al. Inflamm Bowel Dis 2010, doi:10.1002/ ibd.21221

\section{PTH-092 INTERVAL SCANNING WITH MAGNETIC RESONANCE ENTEROGRAPHY DEMONSTRATES RESPONSE TO ANTI- TNF THERAPY AND HAS UTILITY IN REASSESSIMENT OF CROHN'S DISEASE}

doi:10.1136/gutjnl-2013-304907.579

1,2, R Dart, ${ }^{3} \mathrm{~N}$ Griffin, ${ }^{3} \mathrm{~V}$ Goh, ${ }^{3} \mathrm{~K}$ Taylor, ${ }^{3} \mathrm{~S}$ Anderson, ${ }^{3} \mathrm{~J}$ Sanderson, ${ }^{3} \mathrm{P} \mathrm{M}$ Irving. ${ }^{1}$ Gastroenterology, St Thomas' Hospital; '2Diabetes and Nutritional Sciences Division, King's College London; '3St Thomas' Hospital, London, UK

Introduction NICE guidelines mandate yearly reassessment of disease activity for those treated with anti-TNF therapy (ATT). Magnetic resonance enterography (MRE) is established in the assessment of small bowel Crohn's disease, however, there is little data to support its utility in disease monitoring. We examined MRE prior to treatment and after at least 6 months treatment with ATT, observing for radiological remission or change in disease burden.

Methods We identified 27 patients (infliximab $n=23$ adalimumab $\mathrm{n}=4$ ) who underwent pre-treatment and reassessment MRE from a local database of patients treated with ATT. MRE scans were assessed by a consultant radiologist, measuring location of lesions, number of skip lesions, length of affected small bowel and skip lesion wall thickness.

Results Median time to MRE post initiation of ATT was 12 months (range 6-20). All patients were ATT naïve prior to treatment; all but 2 were treated with concomitant immunosuppression. In $63 \%(n=17)$ of patients, there was small bowel disease noted in > 1 location; terminal ileum $74 \%$ (20), distal ileum $37 \%$ (10), mid ileum $22 \%$ (6), proximal ileum 18\% (5), distal jejunum $15 \%$ (4), mid jejunum $4 \%$ (1) and duodenum $4 \%$ (1). In no instances had disease spread to a new location on interval scanning. Total length of involvement $(\mathrm{cm})$ improved post-treatment from median $15 \mathrm{~cm}$ (range 3-50) to $6.8 \mathrm{~cm}(0-33) \mathrm{p}=0.012$, as did length of the dominant lesion $6.5 \mathrm{~cm}(2.5-30)$ vs $3 \mathrm{~cm}(0-30) \mathrm{p}=0.001$. Lesion bowel wall thickness also improved $7 \mathrm{~mm}(4-12)$ vs $5 \mathrm{~mm}(2-10)$ $p=0.0006$. Disease burden, calculated by total stricture length $x$ bowel wall thickness, also improved, 80 (12-400) vs 32 (0-264) $\mathrm{p}=0.001$. Improvement in number of skip lesions per-patient was not significant $2(1-6)$ vs $1(0-5) p=0.2$; in 2 cases the number of skip lesions increased. In no cases was the total length of involvement 